\title{
Universal Design Principles for Cascade Heterojunction Solar Cells with High Fill Factors and Internal Quantum Efficiencies Approaching 100\%
}

\author{
Adam Barito, Matthew E. Sykes, Bingyuan Huang, David Bilby, Bradley Frieberg, \\ Jinsang Kim, Peter F. Green, and Max Shtein*
}

Cascade heterojunction ( $\mathrm{CH})$ ) organic solar cells have recently emerged as an alternative to conventional bulk heterojunctions and series-connected tandems due to their significant promise for high internal quantum efficiency (IQE) and broad spectral coverage. However, $\mathrm{CH}$ ) devices thus far have also exhibited poor fill factor (FF), resulting in minimal enhancements (or even decreases) in power conversion efficiency (PCE) when compared with single heterojunction (SHJ) cells. In this study, the major variables controlling the $\mathrm{CHJ}$ maximum power point and FF are determined using a combinatorial approach. By matching the maximum power point voltage ( $\left.V_{\mathrm{MPP}}\right)$ of the constituent parallel-connected heterojunctions (subjunctions) and minimizing the injection barriers intrinsic to $\mathrm{CH}$ )s, high FF and PCE can be achieved. Optimized $\mathrm{CH}$ ) devices are demonstrated with $>99 \%$ IQE in the interlayer and a $46 \%$ increase in PCE compared to a $\mathrm{SH}$ ) reference $(4.1 \%$ versus $2.8 \%$ ). Devices with a transparent exciton dissociation layer (EDL)/interlayer/acceptor structure are employed, such that each $\mathrm{CH}$ ) has absorption efficiency identical to its interlayer/acceptor $\mathrm{SH}$ ) counterpart. Using these results, a clear map of performance as a function of material parameters is developed, providing straightforward, universal design rules to guide future engineering of molecules and layer architectures for $\mathrm{CH}$ ) organic photovoltaic devices.

substantially lower short-circuit current densities $\left(U_{\mathrm{sc}}\right)$, owing largely to their limited coverage of the solar spectrum and non-radiative losses involving the diffusion and dissociation of strongly bound excitons during the photoconversion process. While the optical absorption length of typical organic materials used for active layers is $\approx 100 \mathrm{~nm}$, the characteristic diffusion length $\left(L_{\mathrm{D}}\right)$ for photogenerated excitons is an order of magnitude lower $(\approx 10 \mathrm{~nm}),{ }^{[2]}$ leading to an efficiency tradeoff with respect to layer thickness. ${ }^{[3]}$

Two major device architectures have been used to circumvent the absorption/ diffusion tradeoff: bulk heterojunctions $(\mathrm{BHJs})^{[4]}$ and vertically stacked, seriesconnected tandem devices..$^{[5,6]}$ In BHJs, the active layers are intermixed to create a spatially distributed heterojunction. With careful morphological control, the size of any donor or acceptor domain within the bulk can be decreased to less than the active materials' $L_{\mathrm{D}}$. Without the thickness limitation by $L_{\mathrm{D}}$, the active layer thickness can be increased to improve absorption efficiency, although this can lead to an

\section{Introduction}

Organic photovoltaics (OPVs) have attracted considerable scientific and technological attention for cost-effective solar energy harvesting with rapid energy payback. ${ }^{[1]}$ However, when compared with their inorganic counterparts, OPVs still exhibit

\footnotetext{
A. Barito, M. E. Sykes, B. Huang, D. Bilby, Prof. J. Kim, Prof. P. F. Green, Prof. M. Shtein

Materials Science and Engineering

University of Michigan

Ann Arbor, MI 48109, USA

E-mail: mshtein@umich.edu

B. Frieberg

Macromolecular Science and Engineering

University of Michigan

Ann Arbor, MI 48109, USA

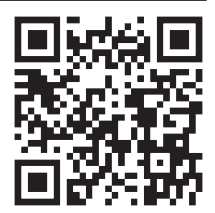

DOI: 10.1002/aenm.201400216 increase in non-geminate recombination. ${ }^{[7]}$ Furthermore, control of the $\mathrm{BHJ}$ active layer morphology remains the primary challenge for both device optimization and materials design. In the case of series tandems, multiple subcells with complementary absorption peaks are used to achieve higher absorption efficiency across the visible spectrum. The latter approach is generalizable, as the subcells in principle can comprise either planar or bulk heterojunctions. However, because tandem devices are connected electrically in series, the resulting device performance is voltage-additive and current-limited by the lowest current of either subcell. ${ }^{[6]}$

A new approach to circumventing the absorption/diffusion tradeoff involves cascade heterojunction $(\mathrm{CHJ})$ devices. ${ }^{\left[{ }^{8-10]}\right.}$ In $\mathrm{CHJs}$, the highest occupied molecular orbital (HOMO) and lowest unoccupied molecular orbital (LUMO) levels of three or more active layers are progressively offset to create multiple energetically cascading heterojunctions within the device. In the simplest case, a planar $\mathrm{CHJ}$ employs a three-layer architecture consisting of a donor/interlayer/acceptor stack: the interlayer 


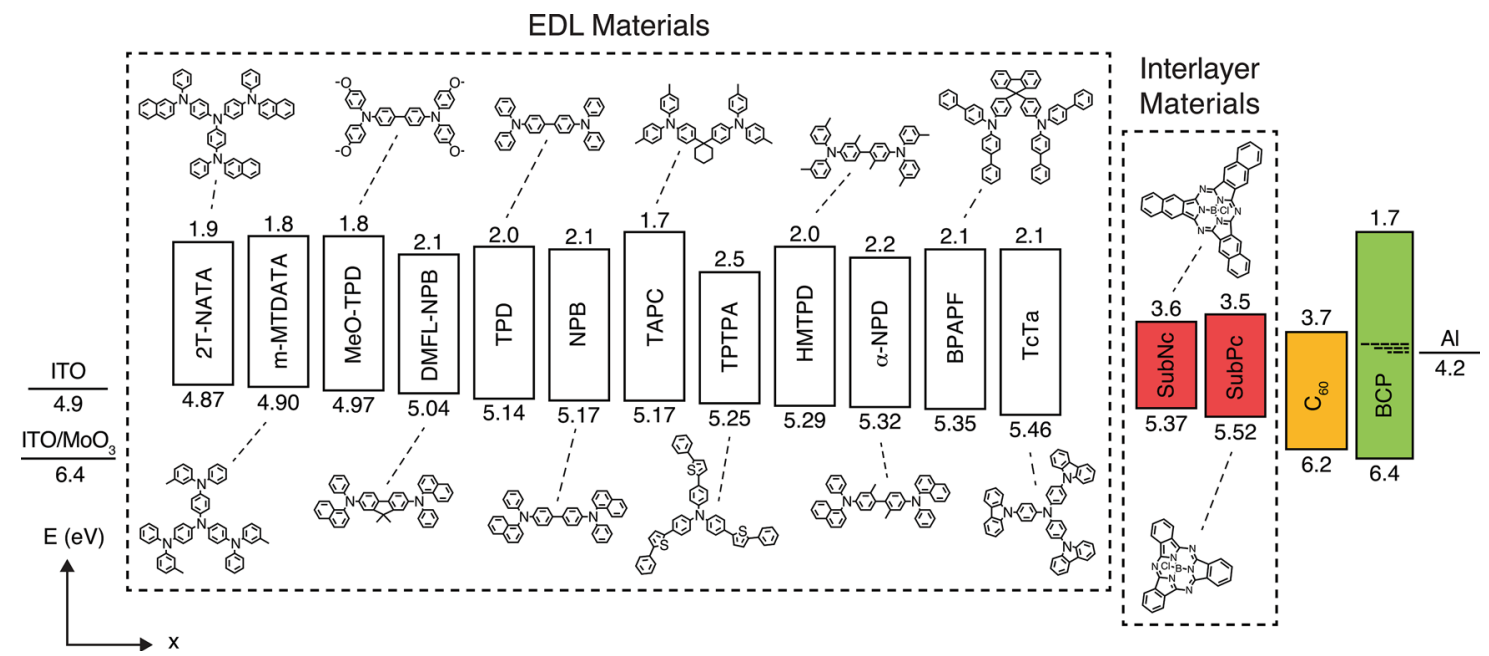

Figure 1. Schematic of energy levels and molecular structures for all materials used in this study. HOMO levels of EDL and interlayer materials were measured using cylic voltammetry and bandgap energies were estimated from the absorption onset. The HOMO level of $\mathrm{C}_{60}$ was taken from literature and it's bandgap energy was estimated from the absorption onset. ${ }^{[16]}$ Energy levels for BCP and the electrodes were taken from literature. ${ }^{[14]}$ The prospective EDL materials were chosen such that their $\mathrm{HOMO}$ levels ranged semicontinously from approximately $4.9 \mathrm{eV}$ to $5.5 \mathrm{eV}$. The two interlayers were chosen based on their differences in $\mathrm{V}_{\mathrm{MPP}}$ when in $\mathrm{SH}$ J configurations with $\mathrm{C}_{60}$.

is sandwiched between two heterojunctions, enabling exciton dissociation on both the donor and acceptor sides, thereby reducing the distance excitons must travel before dissociating. This reduced diffusion distance can substantially increase the internal quantum efficiency (IQE) of the interlayer, resulting in a higher external quantum efficiency (EQE) and overall device $J_{\mathrm{sc}}$. Compared to BHJs, planar devices can offer nearly $100 \%$ charge collection efficiency, more straightforward optimization of optical absorption, and more refined control over individual layer morphologies. ${ }^{[11]}$ Due to the nature of $\mathrm{CHJ}$ device design, it is also possible to broaden spectral coverage by using three (or more) active layers with absorption peaks in nonoverlapping regions of the spectrum, providing an alternative or complementary approach to series tandem configurations. However, efficient charge collection in CHJs does not automatically lead to a good fill factor (FF). ${ }^{[9,10]}$ Indeed, in a previous study we demonstrated a $66 \%$ increase in the IQE and EQE of boron subphthalocyanine chloride (SubPc) by introducing a large bandgap, transparent exciton dissociation layer (EDL) between SubPc and the anode in a planar single heterojunction (SHJ) SubPc/ $\mathrm{C}_{60}$ device. ${ }^{[10]}$ Although the $J_{\mathrm{sc}}$ improved significantly, the overall PCE exhibited only a minimal increase due to a concomitant decrease in FF, leaving open questions as to the fundamental limitations of the $\mathrm{CHJ}$ solar cell architecture.

Here, we perform an extensive, highly systematic study of the EDL/interlayer/acceptor system to probe the underlying mechanisms that cause low FFs in CHJ devices. We use 12 exciton dissociation layers, coupled with SubPc or boron subnaphthalocyanine chloride ( $\mathrm{SubNc}$ ) as interlayers and $\mathrm{C}_{60}$ as the acceptor Figure 1. By definition, the $\mathrm{FF}$ is a simple way of relating $J_{\mathrm{sc}}$ and $V_{\mathrm{oc}}$ to the maximum power point (MPP):

$\mathrm{FF}=\frac{V_{\mathrm{MPP}} J_{\mathrm{MPP}}}{V_{\mathrm{oc}} J_{\mathrm{sc}}}$ where $V_{\mathrm{MPP}}$ and $J_{\mathrm{MPP}}$ are the voltage and current at the MPP, respectively. However, while FF can be a useful metric for describing device performance, it can be imprecise or misleading if both $J_{\mathrm{sc}}$ and $V_{\mathrm{oc}}$ vary between the devices under consideration. We instead focus on the MPP for comparisons between devices with the understanding that if $V_{\mathrm{MPP}}$ and $J_{\mathrm{MPP}}$ are maximized, then FF will also be maximized. For CHJs, we clearly show that the $V_{\text {MPP }}$ is limited by two major factors, both of which can lead to the onset of s-kink behavior in the current-voltage $(J-V)$ characteristics of the devices. First, we demonstrate that the two active heterojunctions (which we term "subjunctions") in the cascade operate electrically in parallel, ${ }^{[10]}$ with the maximum $V_{\mathrm{MPP}}$ of the $\mathrm{CHJ}$ limited by the lowest $V_{\mathrm{MPP}}$ of the two subjunctions. Second, we show that the $V_{\text {MPP }}$ of a $\mathrm{CHJ}$ is further limited by the energy offset between the HOMO levels $\left(\Delta E_{\text {Номо }}\right)$ of the hole transporting donor layer and interlayer. As $\Delta E_{\text {HOMO }}$ increases, the voltage at the maximum power point $\left(V_{\mathrm{MPP}}\right)$ decreases, leading to a lower FF and PCE. We attribute this parasitic effect to the introduction of an energetic charge injection barrier, which results in a space charge buildup within the device and a corresponding decrease in the built-in field. ${ }^{[12,13]}$ Impressively, for optimized devices we observe an increase in the peak IQE of the SubPc and SubNc layers from $38 \%$ and $66 \%$ to $84 \%$ and $>99 \%$, respectively, over reference single heterojunction (SHJ) devices with no EDL. Furthermore, by matching the $V_{\text {MPP }}$ of each subjunction and choosing an EDL with $\Delta E_{\text {Hомо }} \leq 0.2 \mathrm{eV}$, we minimize any losses in $V_{\mathrm{MPP}}$ (and FF) and demonstrate a $46 \%$ enhancement in PCE for a SubNc CHJ over its SHJ reference device.

\section{Results and Discussion}

\subsection{Active Layer Energy Levels and Device Architectures}

Twelve different triphenylamine derivatives were used in this study as EDLs, selected based on their high hole mobilities, 
transparency in the visible spectrum, and HOMO levels varying from $\approx 4.9 \mathrm{eV}$ to $\approx 5.5 \mathrm{eV}$. Figure 1 depicts a schematic energy level diagram and the molecular structure for all materials used. ${ }^{[14-16]}$ In Figure S1 (Supporting Information), we show the absorption coefficients for each material, with only the interlayers and $\mathrm{C}_{60}$ acceptor having absorption peaks in the visible spectral region. The two interlayer materials were chosen primarily due to their different characteristic $V_{\text {MPP }}$ when paired with $\mathrm{C}_{60}$ in a $\mathrm{SHJ}$ configuration. As demonstrated below, the EDL/interlayer $V_{\text {MPP }}$ often limits the CHJ $V_{\text {MPP }}$, so choosing a reference $\mathrm{SHJ}$ with a lower $V_{\text {MPP }}$ can help match the $V_{\text {MPP }}$ between the EDL/interlayer and interlayer/ $\mathrm{C}_{60}$ subjunctions.

$\mathrm{MoO}_{3}$ was used as an anode buffer layer in all $\mathrm{SHJ}$ and $\mathrm{CHJ}$ devices employing SubPc as an active layer. While $\mathrm{MoO}_{3}$ quenches excitons in SubPc and other common OPV materials, ${ }^{[10,17]}$ its high work function is necessary for sustaining the $\approx 1.1 \mathrm{~V}$ open-circuit voltage of $\mathrm{SubPc} / \mathrm{C}_{60}$ devices. Moreover, $\mathrm{MoO}_{3}$ causes virtually no changes to the optical field profiles within the device, unlike other commonly used buffer layers such as poly(ethylenedioxythiophene):poly(styrenesulfonate). ${ }^{[18]}$ Because $\mathrm{MoO}_{3}$ is not required for the lower $V_{\text {oc }}(\approx 0.8 \mathrm{~V})$ of $\mathrm{SubNc} / \mathrm{C}_{60}$ devices, it was not used in $\mathrm{SubNc} / \mathrm{C}_{60} \mathrm{SHJ}$ or EDL/ SubNc/C 60 CHJ devices. $\mathrm{MoO}_{3}$ was used for all EDL/SubNc
SHJ devices to ensure the built-in field did not limit their $V_{\text {oc. }}$. As will be seen below, all trends in device performance were independent of the anode.

\subsection{Electrical Operation of $\mathrm{CHJ}$ Devices}

To understand $\mathrm{CHJ}$ device operation, we must consider photocurrent generation under short-circuit conditions (determined by EQE) as well as the $V_{\text {MPP }}$ and FF limitations of the device under forward bias. Previously, we showed that during operation at zero applied bias (Figure 2c), both subjunctions in a CHJ device act as current sources operating electrically in parallel, with a barrier-free extraction of charge carriers upon exciton dissociation. ${ }^{[10]}$ Since modeling of the EQE in CHJs was demonstrated previously, we leave the majority of its discussion to the Supporting Information, where we provide a more in-depth focus on the comparison between SHJ and CHJ devices.

By treating the subjunctions as acting electrically in parallel, ${ }^{[10]}$ we can fully consider the $J-V$ characteristics of $\mathrm{CHJ}$ devices under forward bias. In series-connected tandem structures, the $J_{\text {MPP }}$ of the complete device will be limited by the lowest $J_{\text {MPP }}$ of its two (or more) subcells. ${ }^{[6]}$ Analogously,

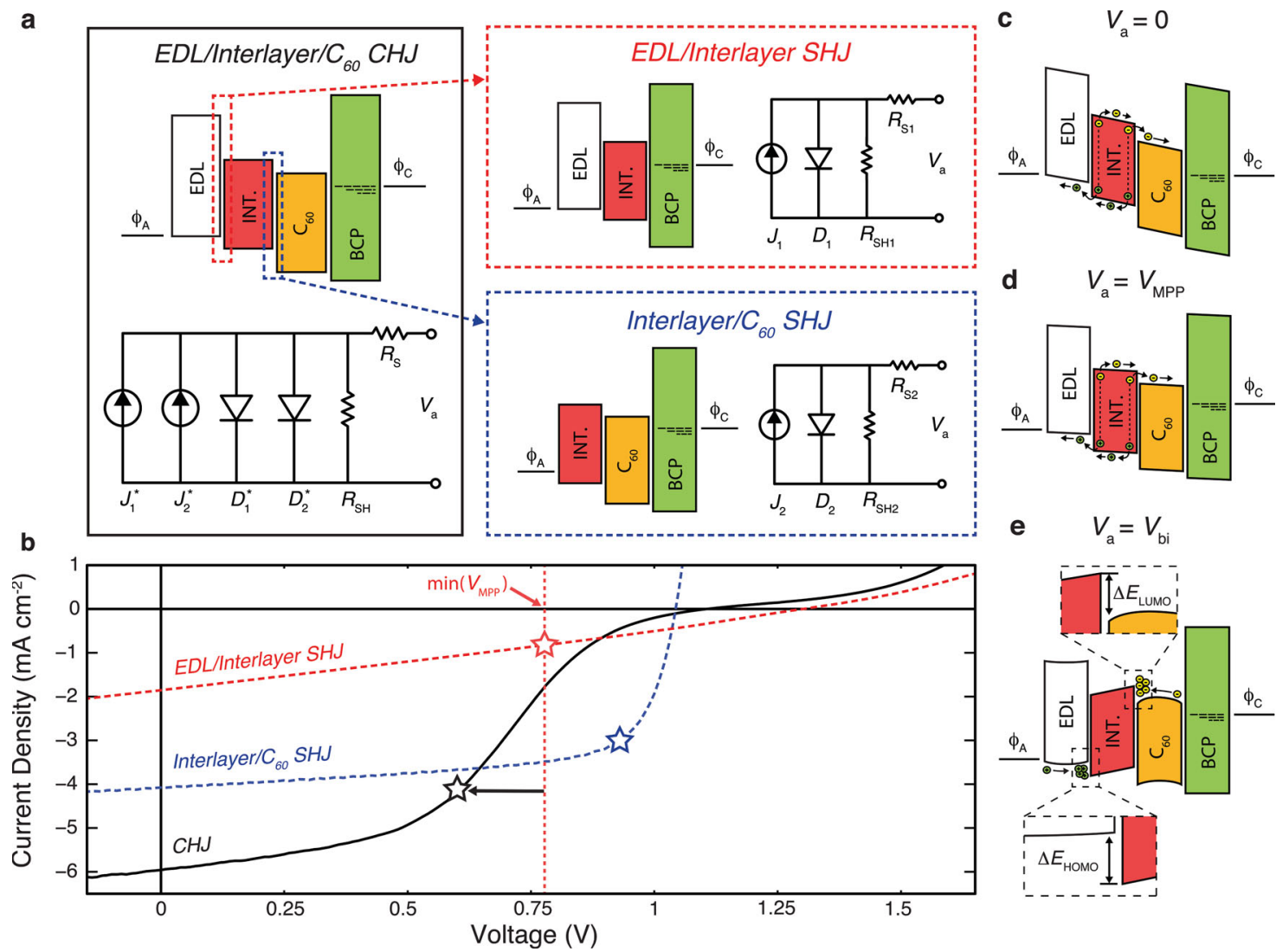

Figure 2. a) Schematic energy level and circuit diagrams for $\mathrm{CHJ}$ devices. The characteristic performance of each subjunction can be estimated by considering the $J-V$ curves of corresponding SHJ devices. b) Characteristic experimental J-V curves of an EDL/interlayer $\mathrm{SHJ}$, an interlayer/ $\mathrm{C}_{60} \mathrm{SHJ}$, and an $\mathrm{EDL} /$ interlayer $/ \mathrm{C}_{60} \mathrm{CH}$ ). The maximum power point for each device is marked by a star. From the two $\mathrm{SH}$ )s, it is clear the $V_{\mathrm{MPP}}$ of the $\mathrm{EDL} /$ interlayer will limit the maximum $V_{M P p}$ of the $\mathrm{CH}$ ) device. Schematic band diagrams of $\left.c\right)$ exciton dissociation in a $\mathrm{CH}$ ) at short-circuit conditions $\left(V_{a}=0\right)$; d) exciton dissociation in a $\mathrm{CHJ}$ at $\mathrm{V}_{\mathrm{MPP}}$, where flat-band conditions have not been met; and e) field inversion at both subjunctions in a $\mathrm{CHJ}$ due to the introduced hole-injection barrier with energy $\Delta E_{\text {номо. }}$ 
the $V_{\text {MPP }}$ of a CHJ device will be limited by the lowest $V_{\text {MPP }}$ of its constituent subjunctions. Due to $\mathrm{CHJ}$ device geometry, it is difficult to measure the $V_{\text {MPP }}$ of each subjunction in situ. However, it is possible to estimate the $V_{\text {MPP }}$ of each subjunction by measuring the $J-V$ characteristics of each subjunction in separate SHJ configurations. These concepts are illustrated in Figure 2a, where equivalent circuit diagrams are provided for each $\mathrm{SHJ}$ device and the $\mathrm{CHJ}$ device comprised of the two corresponding subjunctions. Experimental $J-V$ curves for an EDL/interlayer/acceptor (TAPC/SubPc/C 60 ) system are shown in Figure $2 \mathrm{~b}$. A star shape marks the maximum power point for each device. From this plot, we can see that the $V_{\text {MPP }}$ of the EDL/interlayer subjunction will limit the $V_{\text {MPP }}$ of the $\mathrm{CHJ}$ device. In all experimental results, as discussed further below, the $V_{\mathrm{MPP}}$ of the $\mathrm{CHJ}$ is less than or equal to the lowest $V_{\mathrm{MPP}}$ of the two operating subjunctions.

To minimize losses in $\mathrm{CHJ}$ devices, the $V_{\mathrm{MPP}}$ values of the subjunctions must be closely matched. Previous studies have shown that the most important factors in determining the $V_{\text {oc }}$ of $\mathrm{SHJ}$ devices are the energy of the HOMO-LUMO gap $\left(\Delta E_{\mathrm{HL}}\right)$ and the polaron pair binding energy $\left(E_{\mathrm{B}}\right)$ between the donor and acceptor layers. At best, $V_{\text {MPP }}$ will be limited by the maximum $V_{\text {oc }}$ of the $\mathrm{SHJ}$, as determined by:

$V_{\mathrm{oc}, \max }=\Delta E_{\mathrm{HL}} / q-E_{\mathrm{B}}$

where $q$ is the electron charge. ${ }^{[19]}$ In Figure $3 \mathrm{a}$, we plot $V_{\mathrm{oc}}$ versus $\Delta E_{\mathrm{HL}}$ for all EDL/interlayer $\mathrm{SHJ}$ devices fabricated in this study. As expected, $V_{\mathrm{oc}}$ does increase with larger $\Delta E_{\mathrm{HL}}$, but $E_{\mathrm{B}}$ also appears to increase as $\Delta E_{\mathrm{HL}}$ approaches the interlayer bandgap energy (i.e., $\Delta E_{\mathrm{HOMO}} \approx 0$ ), especially in the case of the EDL/SubNc SHJ devices. This is consistent with experimental findings by Zhang et al., ${ }^{[20]}$ attributable to a linear dependence of the polaron pair separation distance $\left(a_{0}\right)$ on $\Delta E_{\mathrm{HOMO}} \cdot{ }^{[21]}$ Figure $3 \mathrm{~b}$ plots simulated $V_{\mathrm{MPP}}$ values versus $E_{\mathrm{B}}$ for a standard SubPc/C 60 SHJ, with $V_{\text {MPP }}$ values taken from photocurrent curves simulated using the Onsager-Braun model, as detailed in the Supporting Information. ${ }^{[22]}$ Figure $3 \mathrm{~b}$ shows that $V_{\text {MPP }}$ scales linearly with $E_{\mathrm{B}}$, with a $0.1 \mathrm{eV}$ change in $E_{\mathrm{B}}$ causing a $45 \%$ drop in $V_{\mathrm{MPP}}$. Thus we conclude that $\Delta E_{\mathrm{HL}}$ and $E_{\mathrm{B}}$ (or $a_{0}$ ) are critical in matching the $V_{\text {MPP }}$ of each subjunction in the $\mathrm{CHJ}$.

\subsection{Effect of $\Delta E_{\mathrm{HOMO}}$ on $\mathrm{CHJ} V_{\mathrm{MPP}}$}

As demonstrated in Figure $2 \mathrm{~b}$, the $V_{\mathrm{MPP}}$ of a $\mathrm{CHJ}$ can be lower than the $V_{\text {MPP }}$ of either subjunction. To elucidate any other possible loss mechanisms, we investigated the effects of energy level alignment on $\mathrm{CHJ} V_{\text {MPP }}$. It has been well established that injection barriers can lead to s-kink $J-V$ behavior in OPVs, either due to non-ohmic contact at the electrode/donor interface ${ }^{[23]}$ or injection bottlenecks between the $\mathrm{p}$ and $\mathrm{i}$ layers in p-i-n type OPV cells. ${ }^{[12,24]}$ Because cascading energy levels are required for creating multiple heterojunctions, $\mathrm{CHJs}$ inherently contain more injection barriers than $\mathrm{SHJs}$. In $\mathrm{CHJs}$, injected holes and electrons could in principle recombine at either the EDL/interlayer heterojunction or the interlayer/acceptor heterojunction. In practice, however, asymmetric injection barriers and carrier mobilities will force recombination to occur at one of the subjunctions, which will in turn determine the overall diode behavior of the CHJ. ${ }^{[19,25]}$ For devices in this study, and the majority of $\mathrm{CHJs}$ shown previously in literature, phthalocyanines have been used as the interlayer, resulting in a large mismatch between interlayer hole $\left(\mu_{\mathrm{h}}\right)$ and electron $\left(\mu_{\mathrm{e}}\right)$ mobilities. Because $\mu_{\mathrm{h}}>\mu_{\mathrm{e}}$ for most phthalocyanines, recombination of injected charges will preferentially occur at the interlayer/ acceptor interface. Recombination at that interface is favored even more if the electron injection barrier from the acceptor into the interlayer $\left(\Delta E_{\mathrm{LUMO}}\right)$ exceeds the hole injection barrier from the EDL into the interlayer $\left(\Delta E_{\mathrm{HOMO}}\right)$, as is the case for devices in this study with $\Delta E_{\text {HOMO }}<0.2 \mathrm{eV}$ (Figure 2e).
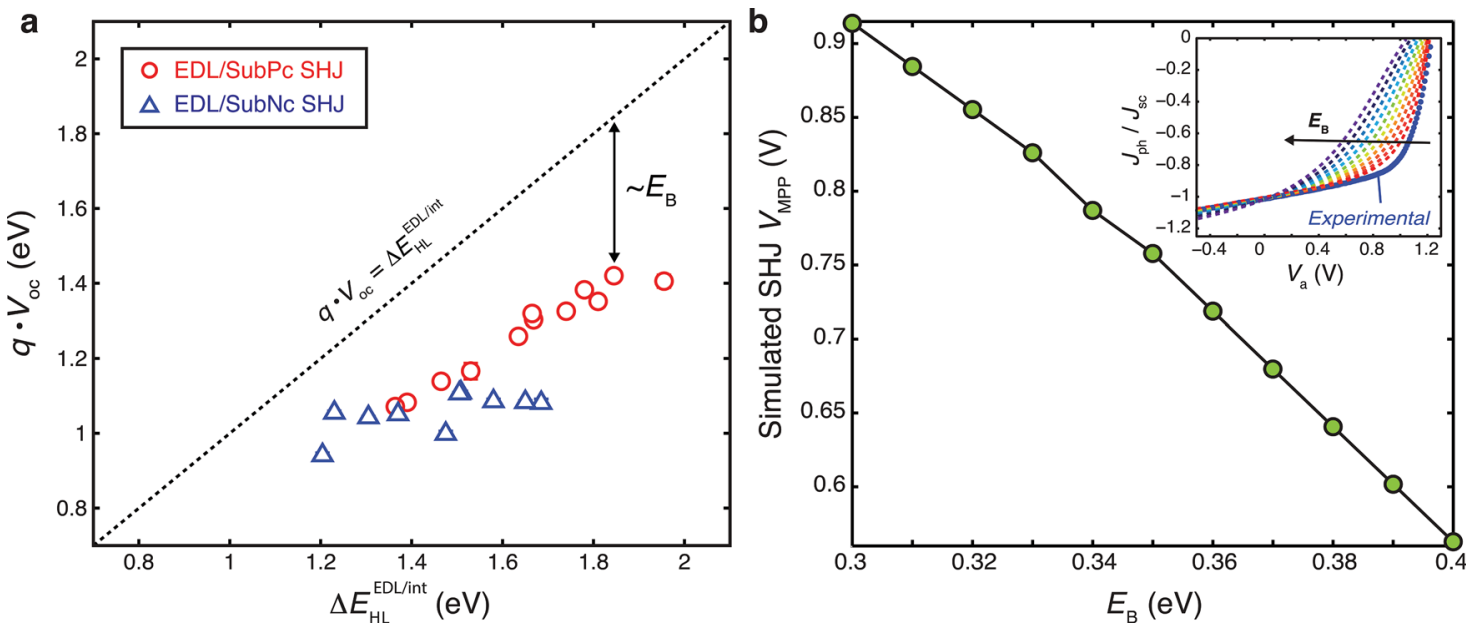

Figure 3. a) $V_{\text {oc }}$ of every EDL/interlayer $\mathrm{SHJ}$ device in this study versus $\Delta E_{\mathrm{HL}}$. Error bars were calculated as one standard deviation of measurements from at least six devices, but are too small to show up in the plot. b) Simulated $V_{M P p}$ for a $S u b P c / C_{60} S H J$ as a function of polaron pair binding energy, $E_{\mathrm{B}}$. Inset: Normalized calculated photocurrent curves vs. applied bias, for varying $E_{\mathrm{B}}$. Simulated photocurrent and $V_{\mathrm{MPP}}$ values were calculated using an Onsager-Braun model, as detailed in the Supporting Information. 
a

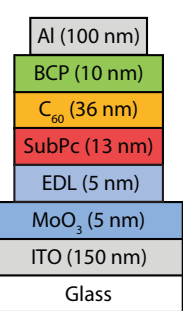

e

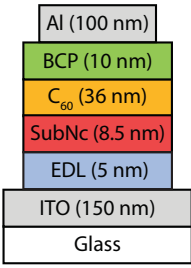

b

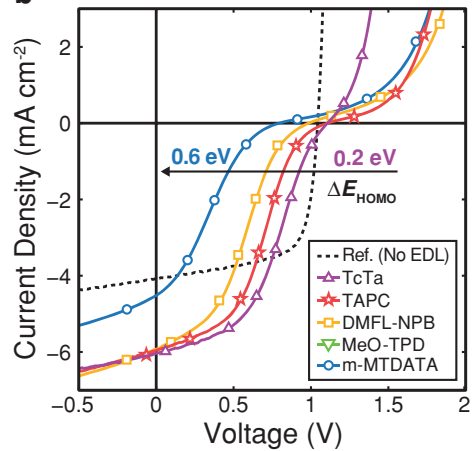

f

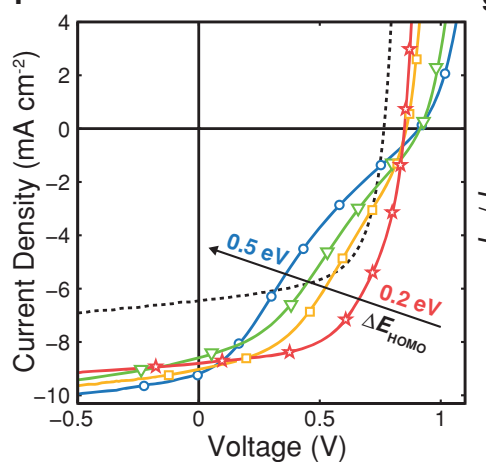

C

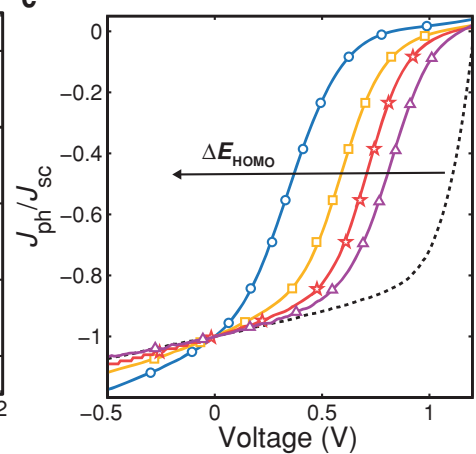

g

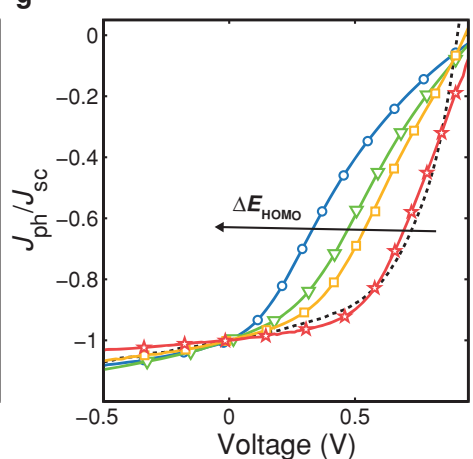

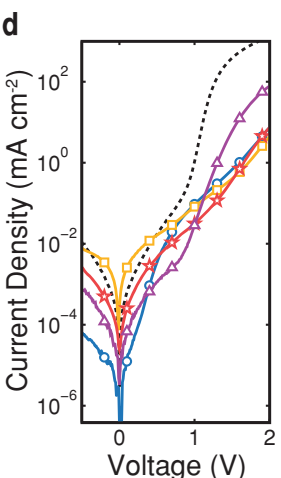

h

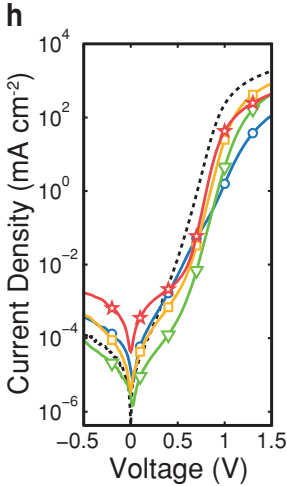

Figure 4. The effect of $\Delta E_{\text {номо }}$ on the $J-V$ performance of $\mathrm{CH}$ J devices. a-d) The device structures, $J-V$, normalized photocurrent, and dark current for devices using SubPc as the interlayer. The device structure for SubPc devices was (all thicknesses in nm) ITO/5 MoO $/ 5 \mathrm{EDL} / 13 \mathrm{SubPc} / 36 \mathrm{C} 60 / 10$ $\mathrm{BCP} / 100 \mathrm{Al}$. e-h) The same data for devices using SubNc as an interlayer. The SubNc device structure was ITO/5 EDL/8.5 SubNc/36 C $60 / 10$ BCP/100 Al. Reference devices with no EDL are represented by dashed black lines.

If recombination occurs at the interlayer/acceptor interface, we must then consider the effect of the HOMO level offset, $\Delta E_{\mathrm{HOMO}}$, introduced by inserting the EDL layer. While this offset is necessary for enabling dissociation at the EDL/interlayer interface and creating a second heterojunction, it also introduces an additional hole injection barrier that can lead to a buildup of charge in the device and a subsequent decrease in the built-in field. ${ }^{[13]}$ At zero bias (Figure 2c), Fermi level alignment in all layers provides band bending that is beneficial to dissociating excitons at each heterojunction; as such, the photocurrent contributions from each subjunction are perfectly additive. As $V_{\mathrm{a}}$ increases $\left(0<V_{\mathrm{a}}<V_{\text {oc }}\right)$, exciton dissociation efficiency $\left(\eta_{\text {Diss }}\right)$ decreases monotonically with the internal field until flat band conditions are reached. The maximum power point will occur at $V_{\mathrm{a}}=V_{\mathrm{MPP}}$, before flat band conditions (Figure 2d). Typically the field inside the active layers is assumed to be nearly constant below $V_{\text {oc }}$, although this is not necessarily the case in $\mathrm{CHJs}$. Tress et al. employed a system using multiple hole transport layers and a transparent interlayer (called a donor layer in the study, as the only photocurrent-producing heterojunction was located at the interlayer/acceptor interface). Using a recursive transport model, $\Delta E_{\mathrm{HOMO}}$ was shown to cause field inversion at the heterojunction (band bending in opposition to exciton dissociation), causing a sharp drop in $\eta_{\text {Diss }}$, shutting off photocurrent production before $V_{\mathrm{oc}}$ and causing s-kink behavior in the $J-V$ curve (Figure 2e). However, in that study, both the HTL and "donor" layers were transparent, meaning that all photocurrent generation came from absorption in the acceptor $\left(\mathrm{C}_{60}\right)$ layer. In this study, we employ $\mathrm{CHJ}$ devices with photocurrent generation occurring at both subjunctions, but expect a similar behavior to occur. To verify, we now experimentally determine the dependence of $\mathrm{CHJ} V_{\mathrm{MPP}}$ on $\Delta E_{\mathrm{HOMO}}$.

In Figure 4 , we show how $\Delta E_{\text {HOMO }}$ can affect $J-V$ performance by varying the material used for the $5 \mathrm{~nm}$ transparent EDL. Figure $4 \mathrm{a}-\mathrm{d}$ and Figure $4 \mathrm{e}-\mathrm{h}$ show $J-V$ curves for $\mathrm{CHJ}$ devices using SubPc and SubNc as the interlayer, respectively. The black dashed line in each plot represents the reference interlayer $/ \mathrm{C}_{60} \mathrm{SHJ}$ device without an EDL. The onset of s-kink behavior is most apparent in Figure $4 \mathrm{c}$ and Figure $4 \mathrm{~g}$, where we normalize the photocurrent for each device to its own $J_{\mathrm{sc}}$. This provides a useful metric for the shape of the device curve regardless of the $J_{\mathrm{sc}}$, and more clearly illustrates that the onset of s-kink behavior in the device is due to field inversion (and resultant shutting down of photocurrent production) at $V_{\mathrm{a}}<V_{\text {oc }}$. Furthermore, in comparing the $J-V$ curves of the devices under no illumination, we note that the dark current at $V_{\text {oc }}$ is $10-100 \times$ lower in the CHJs than in the SHJ reference device without an EDL. Lower dark currents at biases close to $V_{\text {oc }}$ indicate a decrease in recombination of injected charges at the dominant heterojunction, providing further evidence for a buildup of holes at the EDL/interlayer interface. If injected holes are unable to reach the interlayer $/ \mathrm{C}_{60}$ interface, they cannot recombine with injected electrons and contribute to dark current. 
a

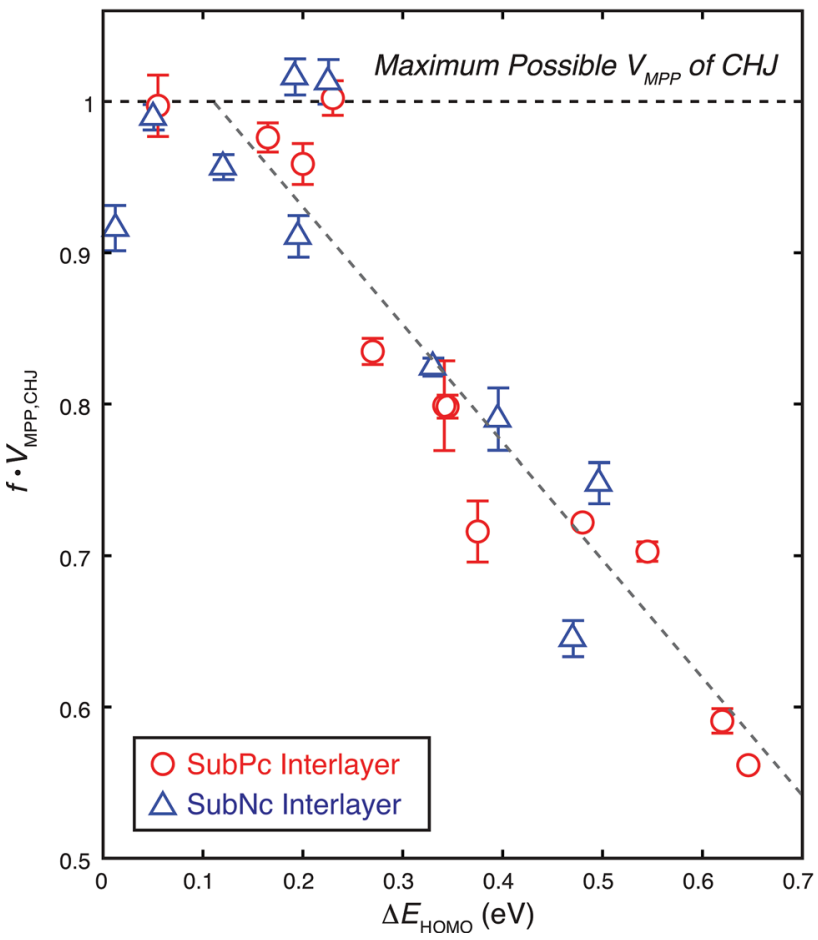

b

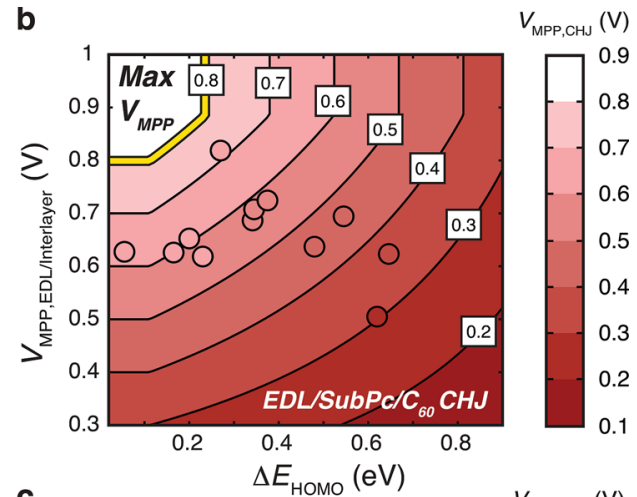

C

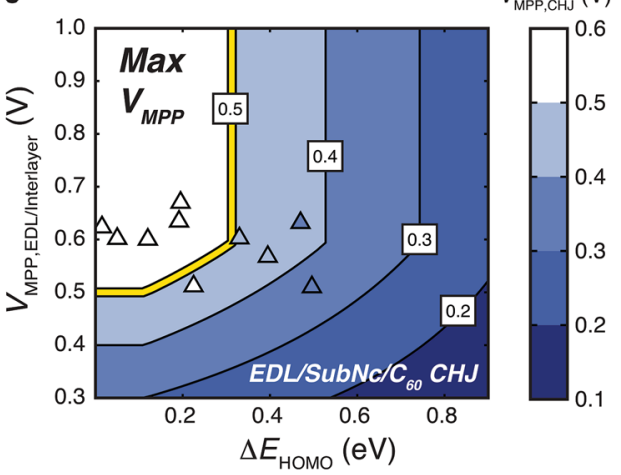

Figure 5. a) A plot of each $\mathrm{CHJ} V_{\text {MPP }}$ normalized by the minimum $V_{\text {MPP }}$ of its constituent subjunctions versus $\Delta E_{\text {HOMO }}$ (as defined in (3)). The dashed horizontal line $=1$ represents the maximum possible $V_{\mathrm{MPP}}$ of the $\mathrm{CH}$ ) based on each subcell. Beyond $\Delta E_{\mathrm{HOMO}} \approx 0.2 \mathrm{eV}$, the $\mathrm{CHJ} V_{\mathrm{MPP}}$ is further lowered due to a decrease in $V_{\text {bi }}$ (and therefore photocurrent) under forward bias. Error bars represent standard deviations calculated from six or more devices. $b, c)$ Contour plots of simulated $V_{M P P}$ for $\mathrm{CHJ}$ devices with SubPc and SubNc interlayers, respectively. $\mathrm{CHJ} V_{M P P}$ is determined by the minimum $V_{M P P}$ of either subjunction and further decreased by $\Delta E_{\mathrm{HO}}$, dictated by the linear fit in (a), as given in (6). Experimental data points (circles and triangles) for $\mathrm{CHJ}$ devices are plotted and colored corresponding to their experimentally determined $V_{\text {MPP. }}$.

\subsection{Dependence of $\mathrm{CHJ} V_{\mathrm{MPP}}$ on $\Delta E_{\mathrm{HOMO}}$ and $V_{\mathrm{MPP}}$ of Subjunctions}

To summarize the combined contributions of field inversion and voltage-limited operation, we measured the $J-V$ performance of all EDL/interlayer and interlayer/ $\mathrm{C}_{60} \mathrm{SHJ}$ devices, extracting the $V_{\text {MPP }}$ for each (performance parameters for all devices can be found in Table S2,S3, Supporting Information). Figure $5 \mathrm{a}$ plots the normalized $V_{\text {MPP }}$ of each $\mathrm{CHJ}$ versus $\Delta E_{\mathrm{HOMO}}$, with the normalization factor $f$ defined as:

$$
f=\frac{1}{\min \left(V_{\mathrm{MPP}}^{\mathrm{EDL} / \text { int }}, V_{\mathrm{MPP}}^{\mathrm{int} / \mathrm{C} 00}\right)}
$$

where $f$ is the inverse of the minimum $V_{\text {MPP }}$ of either subjunction operating in the $\mathrm{CHJ}$. Remarkably, the data collapse onto a universal trend, indicating that for $\Delta E_{\mathrm{HOMO}}<0.2 \mathrm{eV}$, the $\mathrm{CHJ}$ is primarily limited by the lowest subjunction $V_{\mathrm{MPP}}$ and operates purely as a set of parallel diodes. However, for $\Delta E_{\mathrm{HOMO}}>0.2 \mathrm{eV}$, the hole injection barrier becomes significant enough to shut down photocurrent production before $V_{\mathrm{oc}}$, decreasing $V_{\text {MPP }}$ below that of either subjunction. This $0.2 \mathrm{eV}$ threshold is consistent with what has been shown in bilayer organic light-emitting diodes, where efficient hole injection into the electron transport layer occurs only when $\Delta E_{\mathrm{HO} O}$ is less than $0.1-0.3 \mathrm{eV}^{[26]}$ Thus, Figure 5a encompasses the critical parameters that will determine the MPP (and thus PCE) of a $\mathrm{CHJ}$ device. From the plot, we conclude that for a high efficiency CHJ, the $V_{\text {MPP }}$ of each subjunction must be closely matched and $\Delta E_{\text {HOMO }}$ between the EDL and interlayer should be kept below $0.2 \mathrm{eV}$.

We note that the HOMO levels of the EDL and interlayer materials were obtained via cyclic voltammetry on individual materials (detailed in the Experimental Section). Within the devices, however, the HOMO levels and offset energies could conceivably vary due to band bending or intermixing at the active layer interfaces. Therefore, as with the estimation of each subjunction's $V_{\text {MPP }}$ from the $V_{\text {MPP }}$ of its SHJ counterpart, the measured energy levels provide an approximate value that can be used for predicting CHJ device performance. It is likely that the variations in energy levels and $V_{\text {MPP }}$ of each subjunction within the $\mathrm{CHJ}$ account for some of the data spread seen in Figure $5 \mathrm{a}$.

\subsection{Best Device Performance}

In Figure 6, we show the device results for the best CHJs created from the combinatorial study using either a SubPc (solid red lines) or SubNc (solid blue lines) interlayer. Both data sets are compared to the reference interlayer $/ \mathrm{C}_{60} \mathrm{SHJ}$ devices, which are plotted with dashed lines. We note that these results 

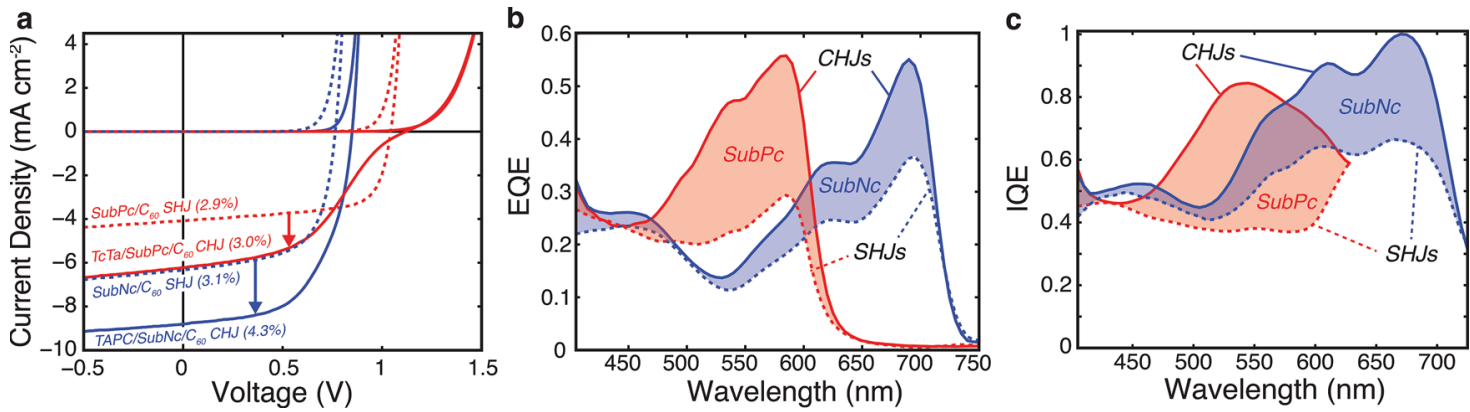

Figure 6. a) $J-V$ curves, b) EQE, and c) IQE of optimized $\mathrm{CH}$ J devices and the corresponding reference SHJ devices with no EDL. Results are shown for cascades with both SubPc and SubNc interlayers. IQE is defined as the experimental EQE divided by the modeled active layer absorption. The increase in $J_{\mathrm{sc}}$ in both $\mathrm{CH}$ )s can be explained by a substantial increase in the IQE and EQE of the interlayers. In the SubNc interlayer, the peak IQE is $>99 \%$. The more pronounced s-kink behavior in the SubPc $\mathrm{CH}$ J is due to the limiting $V_{\mathrm{MPP}}$ of the TcTa/SubPc subjunction.

are consistent with those reported for $\mathrm{SubPc} / \mathrm{C}_{60}$ and $\mathrm{SubNc} /$ $\mathrm{C}_{60}$ devices using other HTL materials. ${ }^{[27,28]}$ Figure 6a shows that the $J_{\mathrm{sc}}$ for both $\mathrm{CHJs}$ is significantly higher than the $J_{\mathrm{sc}}$ of the respective reference devices due to the large increase in EQE (Figure 6b) and IQE (Figure 6c) of the interlayers. Here, we have defined the IQE as:

$$
\operatorname{IQE}(\lambda)=\frac{\mathrm{EQE}(\lambda)}{\eta_{\mathrm{Abs}}(\lambda)}
$$

where $\operatorname{EQE}(\lambda)$ is the experimentally determined external quantum efficiency of the device and $\eta_{\mathrm{Abs}}(\lambda)$ is the absorption of only the active layers at wavelength $\lambda$, as determined by optical modeling (fittings by the optical model are shown in Figure S2, Supporting Information). Impressively, the IQE of the SubNc interlayer within the cascade approaches 100\% ( $>90 \%$ from $650-700 \mathrm{~nm}$, with a peak value of $>99 \%$ ), meaning that nearly all photogenerated excitons in the SubNc are converted to electrical current. Furthermore, the $V_{\text {MPP }}$ of the SubNc CHJ is insensitive to the insertion of a $5 \mathrm{~nm}$ TAPC EDL between the ITO anode and the SubNc layer, while the SubPc CHJ sees a large drop in $V_{\mathrm{MPP}}$, consistent with a prior result. ${ }^{[9]}$ Consequently, the FF of the SubNc/ $\mathrm{C}_{60} \mathrm{SHJ}(62 \%)$ is largely maintained in the TAPC/SubNc/ $\mathrm{C}_{60} \mathrm{CHJ}(58 \%)$, whereas the $\mathrm{FF}$ of the $\mathrm{TcTa} / \mathrm{SubPc} / \mathrm{C}_{60} \mathrm{CHJ}$ (44\%) decreases significantly compared to the SubPc/ $\mathrm{C}_{60} \mathrm{SHJ}(67 \%)$. This makes empirical sense, considering the $V_{\text {MPP }}$ of each SubPc subjunction $(0.63 \pm 0.01 \mathrm{~V}$ for the TcTa/SubPc SHJ and $0.89 \pm 0.01 \mathrm{~V}$ for the SubPc/C 60 $\mathrm{SHJ})$, with the $\mathrm{TcTa} / \mathrm{SubPc}$ subjuncton limiting the overall $V_{\text {MPP }}$ of the CHJ to $0.63 \pm 0.01 \mathrm{~V}$. On the other hand, the $V_{\text {MPP }}$ of each SubNc subjunction is closely matched $(0.63 \pm 0.01 \mathrm{~V}$ for the TAPC/SubNc SHJ and $0.59 \pm 0.01 \mathrm{~V}$ for the SubNc/C 60 $\mathrm{SHJ}$ ), leading to a $\mathrm{CHJ} V_{\mathrm{MPP}}=0.60 \pm 0.01 \mathrm{~V}$.

\section{6. $V_{\text {oc }}$ Limitations in $\mathrm{CH}$ J Devices}

Recently, Cnops et al. suggested that the $V_{\text {oc }}$ of $\mathrm{CHJs}$ should be limited by the energy levels of the outermost active layers. [29] This limitation on the $V_{\text {oc }}$ would occur due to the additional losses in energy as the free charges are extracted from the device. In Figure 7, we plot the $V_{\text {oc }}$ of each $\mathrm{CHJ}$ versus $\Delta E_{\mathrm{HL}}^{\mathrm{EDL} / \mathrm{C} 60}$ (the difference in energy between the HOMO level of the EDL and the LUMO level of the $\mathrm{C}_{60}$ layer), and indeed we see that the $V_{\text {oc }}$ can be limited for a small enough $\Delta E_{\mathrm{HL}}^{\mathrm{EDL} / \mathrm{C6} 0}$. In the CHJ devices with a SubPc interlayer, we observe a crossover point at $\Delta E_{\mathrm{HL}}^{\mathrm{EDL} / \mathrm{C} 60} \approx 1.45 \mathrm{eV}\left(\Delta E_{\mathrm{HOMO}} \approx 0.35 \mathrm{eV}\right)$, above which the $V_{\mathrm{oc}}$ remains relatively constant, and below which the $V_{o c}$ decreases monotonically with decreasing $\Delta E_{\mathrm{HL}}^{\mathrm{ED} / \mathrm{C} 60}$. A similar transition is inferred at $\approx 1.18 \mathrm{eV}\left(\Delta E_{\text {HOMO }} \approx 0.48 \mathrm{eV}\right)$ for devices with a SubNc interlayer, however the limited data below this value makes it more approximate. Critically, any limitations in $V_{\text {oc }}$ only occur for very small $\Delta E_{\mathrm{HL}}^{\mathrm{EDL} / \mathrm{C} 60}$ values. Conversely, for larger $\Delta E_{\mathrm{HL}}^{\mathrm{EDL} / \mathrm{C60}}$ (smaller $\Delta E_{\mathrm{HOMO}}$ ) values, the $\mathrm{CHJ}$ devices actually exhibit an increase in $V_{\text {oc }}$ compared to the reference interlayer $/ \mathrm{C}_{60} \mathrm{SHJ}$, which we attribute to a decrease in dark current (Figure 4d,h). The black dotted line in Figure 7 represents:

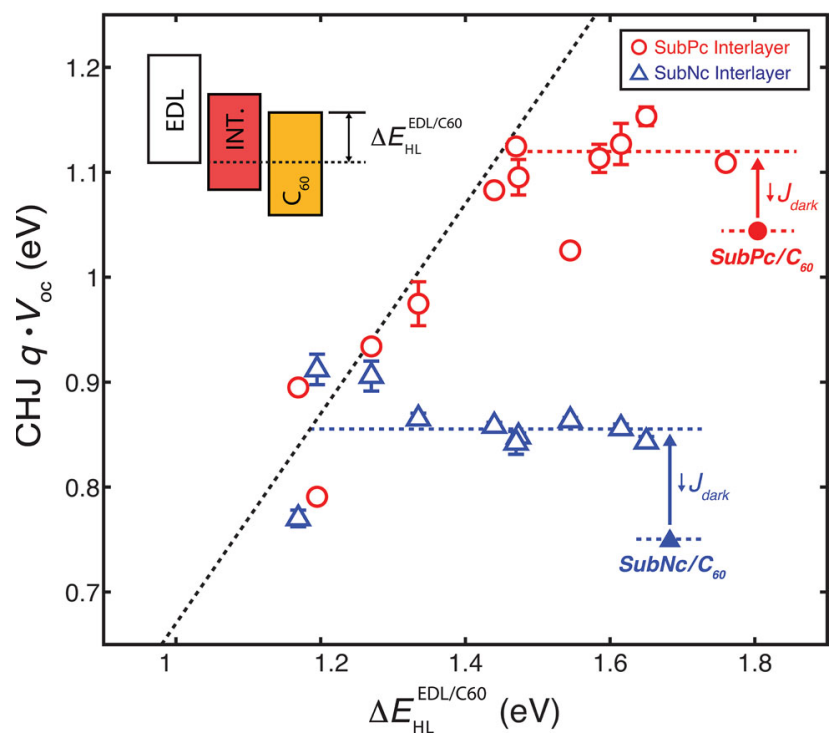

Figure 7. A plot of $q V_{o c}$ for each $C H$ ) device versus $\Delta E_{H L}^{E D L / C 60}$ (the difference in energy between the HOMO and LUMO levels of the EDL and $C_{60}$ layers, respectively). The $V_{o c}$ of the $\mathrm{CH}$ J devices increases initially upon insertion of an EDL due to a decrease in the dark current. As $\Delta E_{\mathrm{HL}}^{\mathrm{EDL} / \mathrm{C} 60}$ decreases, the $V_{o c}$ of the $\mathrm{CH}$ )s remains relatively constant until it becomes limited by $\Delta E_{\mathrm{HL}}^{\mathrm{EDL} / \mathrm{C60}}-E_{\mathrm{B}}$. The diagonal black dotted line represents $\Delta E_{\mathrm{HL}}^{\mathrm{EDL} / \mathrm{C60}}-0.3 \mathrm{eV}$, indicating a binding energy of $0.3 \mathrm{eV}$ (consistent with the fitted $E_{\mathrm{B}}$ in the Supporting Information). 
$q V_{\mathrm{oc}}=\Delta E_{\mathrm{HL}}^{\mathrm{EDL} / \mathrm{C} 60}-0.3 \mathrm{eV}$

indicating that the maximum possible $V_{\text {oc }}$ of the CHJs is limited by $\Delta E_{\mathrm{HL}}^{\mathrm{EDL} / \mathrm{C60}}$ and an effective binding energy of $\approx 0.3 \mathrm{eV}$, consistent with our calculated $E_{\mathrm{B}}$ of the $\mathrm{SubPc} / \mathrm{C}_{60}$ and $\mathrm{SubNc} /$ $\mathrm{C}_{60}$ heterojunctions (Supporting Information). Since a majority of photocurrent in the $\mathrm{CHJ}$ is generated at the interlayer $/ \mathrm{C}_{60}$ interface, ${ }^{[10]}$ it is not surprising that the effective $E_{\mathrm{B}}$ of the $\mathrm{CHJ}$ is close to that of the interlayer $/ \mathrm{C}_{60}$ subjunction.

As demonstrated by the EDL/SubNc/C 60 devices, $\mathrm{CHJs}$ with interlayer/acceptor subjunctions exhibiting high recombination losses can employ donor layers with a larger $\Delta E_{\mathrm{HOMO}}$ before $V_{\mathrm{oc}}$ begins to drop. However, as we have already established that $\Delta E_{\mathrm{HOMO}}$ should be kept to less than $0.2 \mathrm{eV}$ to minimize charge injection barriers in the devices, properly designed $\mathrm{CHJs}$ will not be voltage-limited. Instead, $\mathrm{CHJ}$ operation can substantially reduce recombination losses and bring the $V_{\text {oc }}$ closer to the theoretical maximum.

\subsection{Design Rules for $\mathrm{CHJ}$ Devices}

The results presented in this study can guide future $\mathrm{CHJ}$ device design, principally dictating that $\Delta E_{\mathrm{HOMO}}$ be less than $0.2 \mathrm{eV}$ and the polaron pair binding energy be minimized for the EDL/ interlayer interface. It is also possible now to screen materials systems for their utility in $\mathrm{CHJ}$ configurations. In Figure 5b,c, we extrapolate the relationship shown in Figure 5a to provide contour plots for predicting the $V_{\mathrm{MPP}}$ of an EDL/SubPc/C 60 $\mathrm{CHJ}$ (Figure $5 \mathrm{~b}$ ) and an $\mathrm{EDL} / \mathrm{SubNc} / \mathrm{C}_{60} \mathrm{CHJ}$ (Figure $5 \mathrm{c}$ ) as a function of EDL/interlayer $V_{\mathrm{MPP}}$ and $\Delta E_{\mathrm{HOMO}}$. A linear fit of the universal trend in Figure 5a produces a general equation:

$$
f V_{\mathrm{MPP}, \mathrm{CHJ}}=-\left(0.78 e V^{-1}\right)\left(\Delta E_{\text {НОМО }}\right)+1.08
$$

with the caveat that the cascade $V_{\text {MPP }}$ will not exceed the $V_{\text {MPP }}$ of either subjunction. We note that (6) implies no dependence of $V_{\text {MPP }}$ on other material properties such as charge mobility. In the Supporting Information, we consider such charge mobility effects and in fact show a strong correlation between HOMO level and hole mobility. However, we see no apparent dependence of $\mathrm{CHJ} V_{\mathrm{MPP}}$ on EDL layer thicknesses, and thus conclude that any effects due to mobility are negligible or secondary to the injection barrier introduced by $\Delta E_{\mathrm{HOMO}}$. As an aside, the apparent relationship between HOMO level and hole mobility for these materials warrants further investigation, as it could provide further insight into previous studies with similar systems where changes in device performance were attributed primarily to variations in the hole mobility of the HTL. ${ }^{[27,30]}$

Finally, from comparing the two contour plots (Figure 5b,c), we can see that a much lower EDL/interlayer $V_{\text {MPP }}$ is required to achieve maximum $V_{\mathrm{MPP}}$ in the SubNc $\mathrm{CHJ}$ as compared to the SubPc CHJ. In many cases, the simplest route to a highperformance $\mathrm{CHJ}$ device may be choosing a base device system with higher $J_{\mathrm{sc}}$ and lower $V_{\mathrm{oc}}$ or $V_{\mathrm{MPP}}$. By "trading" $J_{\mathrm{sc}}$ for $V_{\mathrm{MPP}}$, the PCE of the reference SHJ device can remain high, while lowering the required $V_{\mathrm{MPP}}$ of the introduced subjunction in the CHJ.

\section{Conclusions}

We have shown that $\mathrm{CHJ}$ architectures are viable options for high-efficiency planar OPVs, primarily due to their nearly $100 \%$ IQE within the interlayer. To ensure high fill factor, the $V_{\text {MPP }}$ of each subjunction must be matched and the HOMO level offset between the EDL and interlayer should be $<0.2 \mathrm{eV}$. Using these proposed design rules, we demonstrated a $46 \%$ increase in the power conversion efficiency of a SubNc/C ${ }_{60}$ planar device by introducing a transparent EDL between SubNc and the ITO anode (from $2.8 \% \pm 0.2 \%$ to $4.1 \% \pm 0.2 \%$ ). By introducing the $5 \mathrm{~nm}$ layer of TAPC, the IQE of the SubNc layer increased from $66 \%$ to $>99 \%$ at its peak, while the high fill factor of the SubNc/C_60 SHJ was largely maintained.

While the PCE was significantly enhanced in properly designed CHJs, $J_{\text {sc }}$ could be improved further through increased active layer absorption. Because the presence of two heterojunctions relaxes the tradeoff between absorption and exciton diffusion, the interlayer thickness can be increased to maximize absorption. Some materials are more suitable for this than others; Verreet et al. recently showed that replacing $\mathrm{C}_{60}$ with hexachlorinated boron subphthalocyanine chloride allowed the SubNc layer thickness to increase upwards of $20 \mathrm{~nm} .{ }^{[28]}$ Furthermore, by using a smaller bandgap material in place of the transparent EDL to increase spectral coverage, device $J_{\text {sc }}$ should increase without any additional drop in $V_{\text {oc }}, V_{\text {MPP }}$, or FF. Because the CHJ devices have such high IQE, they are also ideal candidates for use as sub-cells in series-connected tandems, potentially allowing for high efficiency OPVs comprising six or more active layers with complementary absorption peaks.

\section{Experimental Section}

Energy Levels: HOMO levels for all interlayer and EDL materials were measured via cyclic voltammetry. Each material was dropcast from chloroform onto a $3 \mathrm{~mm}$ diameter glassy carbon working electrode. Using $0.1 \mathrm{M}$ tetrabutylammonium hexafluorophosphate in acetonitrile as an electrolyte, samples were scanned at a rate of $0.1 \mathrm{~V} \mathrm{~s}^{-1}$ relative to an $\mathrm{Ag} / \mathrm{AgNO}_{3}$ reference electrode with a Pt wire counter electrode. Scans were normalized to the onset of oxidation of ferrocene, taken as $-4.8 \mathrm{eV}$. The bandgap was estimated from the onset of absorption, and the LUMO level was calculated by adding the bandgap to the HOMO level.

Device Fabrication: Devices were deposited on commercially available ITO (Delta Technologies, $150 \mathrm{~nm}$ thick, $R_{\mathrm{s}}<15 \Omega / \square$ ). Substrates were cleaned via heated $\left(40^{\circ} \mathrm{C}\right)$ sonication in detergent, water, acetone, trichloroethylene, and isopropanol, followed by boiling in isopropanol and 10 minutes of ultraviolet/ozone treatment to remove carbon residues and increase the anode work function. Device layers were deposited via vacuum thermal evaporation (VTE) using an Ångstrom AMOD deposition chamber. Fabrication and $J-V$ testing was performed in a glovebox filled with an inert nitrogen environment $\left(<1 \mathrm{ppm} \mathrm{O}_{2}\right.$ and $\mathrm{H}_{2} \mathrm{O}$ ). To minimize degradation in atmosphere during testing, devices were simultaneously deposited on three substrates, so that one of each could be used for testing J-V, EQE, and absorption. Only samples for $\mathrm{EQE}$ and absorption measurements were exposed to atmosphere. For EQE and $J-V$ testing, aluminum island electrodes were deposited through a shadow mask with a diameter of $1 \mathrm{~mm}$. All device areas were measured using a Carl Zeiss Scope A.1 optical microscope and included explicitly in calculating $J_{s c}, E Q E, I Q E$, and PCE. All organic materials were purchased from Luminescence Technology Corp. and deposited with no further purification. SubPc, SubNc, BCP, and all EDL materials (>99\%) and $\mathrm{C}_{60}(>99.5 \%)$ were sublimed grade. $\mathrm{MoO}_{3}(>99.99 \%)$ was purchased from Sigma Aldrich and Al (99.9\%) was purchased from Alfa Aesar. 
Device Characterization: Device $J-V$ curves were recorded using an HP 4156B precision semiconductor parameter analyzer. The cells were illuminated with a Newport solar simulator (model\# 91191-1000) calibrated to AM1.5 (100 $\left.\mathrm{mW} \mathrm{cm} \mathrm{cm}^{-2}\right)$ using an NREL Si reference cell (Model PVM233 KG5). EQE was measured by directing a collimated beam of optically chopped light $(185 \mathrm{~Hz})$ from a halogen lamp coupled to a Newport $1 / 8 \mathrm{~m}$ monochromator (5 $\mathrm{nm}$ FWHM) incident on the sample. The photocurrent was measured using a Stanford Research Systems SR530 lock-in amplifier and compared to the output from a calibrated Si photodiode. The spectrum of the solar simulator was measured with an Ocean Optics USB2000 spectrometer and convoluted with the experimental EQE to determine the spectral mismatch factor for each device with respect to the AM1.5G spectrum. ${ }^{[31]}$ All mismatch factors were determined to be $1 \pm 0.05$. Absorption in the completed devices was measured in reflection mode using a Perkin Elmer Lambda 750 UV/Vis/NIR spectrometer at an incidence angle of $7.5^{\circ}$. The absorption spectrum for each device was then compared to a transfer matrix optical model to confirm device layer thicknesses. IQE was calculated by dividing experimental EQE by modeled active layer absorption at each wavelength at normal incidence.

Optical Properties of Materials: The thicknesses and optical properties of all materials were measured using a variable angle spectroscopic ellipsometer (M-2000, J.A. Woollam Co.). Measurements were performed in both transmission mode and reflection mode at angles of $55^{\circ}, 65^{\circ}$, and $75^{\circ}$ for each of the materials on a glass substrate. The film thickness and surface roughness were first determined by fitting the acquired ellipsometric angles $\Delta$ and $\Psi$ to a Cauchy model over the wavelength range in which the material is transparent. The refractive index values were then determined by fixing the film thickness as well as surface roughness and parameterizing the material as a B-Spline layer. The wavelength range was gradually increased, in increments of $0.1 \mathrm{eV}$, until it included the entire measured spectral range. The resultant values were then verified to be Kramers-Kronig (KK) consistent.

Mobility Measurements: For hole mobility measurements, samples were fabricated with the structure ITO/PEIE $(10 \mathrm{~nm}) / \operatorname{EDL}(800 \mathrm{~nm}) / \mathrm{Au}$ $(80 \mathrm{~nm})$. PEIE (0.4 wt\% in methoxyethanol) was spin-coated at 5000 rpm for $60 \mathrm{~s}$ and subsequently baked at $100{ }^{\circ} \mathrm{C}$ for $10 \mathrm{~min}$ prior to VTE deposition of the EDL at $1 \AA \mathrm{s}^{-1}$. Circular gold contacts were deposited at $1 \AA \mathrm{s}^{-1}$ and defined by a shadow mask. Time-of-flight measurements were performed using a nitrogen laser (VSL337 from Newport) with a wavelength of $\lambda=337.1 \mathrm{~nm}$, an intensity per pulse of $\approx 120 \mu \mathrm{J}$, and a pulse duration less than $4 \mathrm{~ns}$, for photogeneration of charge carriers in the films (illuminated through the ITO substrate). A Keithley 2400 SourceMeter was used to apply constant voltage over devices, with the ITO cathode under positive bias to prevent charge injection. The current transients were then amplified using a FEMTO DLPCA-200 low noise current amplifier and recorded with a Tektronix TDS3052C digital oscilloscope.

\section{Supporting Information}

Supporting Information is available from the Wiley Online Library or from the author.

\section{Acknowledgements}

This work was supported by the U.S. Department of Energy (DOE), Office of Basic Energy Sciences, as part of the Center for Solar and Thermal Energy Conversion, an Energy Frontier Research Center (Award No. DE-SC0000957). A.B. thanks the NSF for partial support of this work via the National Science Foundation Graduate Student Research Fellowship under Grant No. 1256260. This article was modified after online publication. Errors in the labels in figure 2 were corrected.

Received: February 5, 2014

Revised: March 24, 2014

Published online: May 3, 2014
[1] a) S. E. Gledhill, B. Scott, B. A. Gregg, J. Mater. Res. 2011, 20, 3167; b) S. B. Darling, F. You, RSC Adv. 2013, 3, 17633; c) C. J. Brabec, Sol. Energy Mater. Sol. Cells 2004, 83, 273.

[2] a) P. Peumans, A. Yakimov, S. R. Forrest, J. Appl. Phys. 2003, 93, 3693; b) W. Chen, M. P. Nikiforov, S. B. Darling, Energy Environ. Sci. 2012, 5, 8045 .

[3] a) C. W. Tang, Appl. Phys. Lett. 1986, 48, 183; b) B. A. Gregg, J. Phys. Chem. B 2003, 107, 4688.

[4] a) G. Yu, J. Gao, J. C. Hummelen, F. Wudl, A. J. Heeger, Science 1995, 270, 1789; b) G. Chen, H. Sasabe, Z. Wang, X. F. Wang, Z. Hong, Y. Yang, J. Kido, Adv. Mater. 2012, 24, 2768; c) R. Pandey, Y. Zou, R. J. Holmes, Appl. Phys. Lett. 2012, 101, 033308; d) J. J. M. Halls, C. A. Walsh, N. C. Greenham, E. A. Marseglia, R. H. Friend, S. C. Moratti, A. B. Holmes, Nature 1995, 376, 498.

[5] A. Yakimov, S. R. Forrest, Appl. Phys. Lett. 2002, 80, 1667.

[6] B. E. Lassiter, C. Kyle Renshaw, S. R. Forrest, J. Appl. Phys. 2013, 113, 214505.

[7] R. Pandey, R. J. Holmes, Appl. Phys. Lett. 2012, 100, 083303.

[8] a) G. Zhang, W. Li, B. Chu, L. Chen, F. Yan, J. Zhu, Y. Chen, C. S. Lee, Appl. Phys. Lett. 2009, 94, 143302; b) M. Sim, J. S. Kim, C. Shim, K. Cho, Chem. Phys. Lett. 2013, 557, 88; c) T. D. Heidel, D. Hochbaum, J. M. Sussman, V. Singh, M. E. Bahlke, I. Hiromi, J. Lee, M. A. Baldo, J. Appl. Phys. 2011, 109, 104502; d) Z. R. Hong, R. Lessmann, B. Maennig, Q. Huang, K. Harada, M. Riede, K. Leo, J. Appl. Phys. 2009, 106, 064511.

[9] K. Cnops, B. P. Rand, D. Cheyns, P. Heremans, Appl. Phys. Lett. 2012, 101, 143301.

[10] A. Barito, M. E. Sykes, D. Bilby, J. Amonoo, Y. Jin, S. E. Morris, P. F. Green, J. Kim, M. Shtein, J. Appl. Phys. 2013, 113, 203110.

[11] A. Foertig, A. Wagenpfahl, T. Gerbich, D. Cheyns, V. Dyakonov, C. Deibel, Adv. Energy Mater. 2012, 2, 1483.

[12] W. Tress, K. Leo, M. Riede, Adv. Funct. Mater. 2011, 21, 2140.

[13] D. Cheyns, J. Poortmans, P. Heremans, C. Deibel, S. Verlaak, B. Rand, J. Genoe, Phys. Rev. B 2008, 77.

[14] a) A. Kahn, N. Koch, W. Gao, J. Polym. Sci. Part B: Polym. Phys. 2003, 41, 2529; b) M. T. Greiner, M. G. Helander, W. M. Tang, Z. B. Wang, J. Qiu, Z. H. Lu, Nat. Mater. 2012, 11, 76.

[15] Z.-L. Guan, J. B. Kim, H. Wang, C. Jaye, D. A. Fischer, Y.-L. Loo, A. Kahn, Org. Electron. 2010, 11, 1779.

[16] M. S. Dresselhaus, G. Dresselhaus, P. C. Eklund, Science of Fullerenes and Carbon Nanotubes, Academic, Waltham, MA 1996.

[17] X. Xiao, J. D. Zimmerman, B. E. Lassiter, K. J. Bergemann, S. R. Forrest, Appl. Phys. Lett. 2013, 102, 073302.

[18] Y.-C. Tseng, A. U. Mane, J. W. Elam, S. B. Darling, Sol. Energy Mater. Sol. Cells 2012, 99, 235.

[19] B. Rand, D. Burk, S. Forrest, Phys. Rev. B 2007, 75.

[20] M. Zhang, H. Wang, C. W. Tang, Appl. Phys. Lett. 2010, 97, 143503.

[21] P. Peumans, S. R. Forrest, Chem. Phys. Lett. 2004, 398, 27.

[22] a) L. Onsager, Phys. Rev. 1938, 54, 554; b) C. L. Braun, J. Chem. Phys. 1984, 80, 4157.

[23] a) A. Wagenpfahl, D. Rauh, M. Binder, C. Deibel, V. Dyakonov, Phys. Rev. B 2010, 82; b) M. Glatthaar, M. Riede, N. Keegan, K. Sylvester-Hvid, B. Zimmermann, M. Niggemann, A. Hinsch, A. Gombert, Sol. Energy Mater. Sol. Cells 2007, 91, 390; c) M. R. Lilliedal, A. J. Medford, M. V. Madsen, K. Norrman, F. C. Krebs, Sol. Energy Mater. Sol. Cells 2010, 94, 2018.

[24] C. Uhrich, D. Wynands, S. Olthof, M. K. Riede, K. Leo, S. Sonntag, B. Maennig, M. Pfeiffer, J. Appl. Phys. 2008, 104, 043107.

[25] a) N. C. Giebink, B. E. Lassiter, G. P. Wiederrecht, M. R. Wasielewski, S. R. Forrest, Phys. Rev. B 2010, 82; b) N. C. Giebink, G. P. Wiederrecht, M. R. Wasielewski, S. R. Forrest, Phys. Rev. B 
2010, 82; c) C. W. Schlenker, M. E. Thompson, Chem. Commun. 2011, 47, 3702.

[26] a) J. Yang, J. Shen, J. Phys. D 2000, 33, 1768; b) N. Matsumoto, C. Adachi, J. Phys. Chem. C 2010, 114, 4652; c) F. Liu, P. Paul Ruden, I. H. Campbell, D. L. Smith, Appl. Phys. Lett. 2011, 99, 123301.

[27] C. Kulshreshtha, G. W. Kim, R. Lampande, D. H. Huh, M. Chae, J. H. Kwon, J. Mater. Chem. A 2013, 1, 4077.
[28] B. Verreet, K. Cnops, D. Cheyns, P. Heremans, A. Stesmans, G. Zango, C. G. Claessens, T. Torres, B. P. Rand, Adv. Energy Mater. 2014, DOI: 10.1002/aenm.201301413.

[29] K. Cnops, B. P. Rand, D. Cheyns, B. Verreet, M. A. Empl, P. Heremans, Nat. Commun. 2014, 5, 3406.

[30] C. Kulshreshtha, J. W. Choi, J.-k. Kim, W. S. Jeon, M. C. Suh, Y. Park, J. H. Kwon, Appl. Phys. Lett. 2011, 99, 023308.

[31] C. H. Seaman, Sol. Energy 1982, 29, 291. 